\title{
Evaluation of Receiver Operating Characteristic Curves in a Microwave Apparatus for Breast Lesions Detection
}

\author{
Gianluigi Tiberi ${ }^{1}$, Lorenzo Sani ${ }^{2}$, Alessandro Vispa ${ }^{2}$, Martina Paoli ${ }^{2}$, \\ Alessandra Bigotti ${ }^{2}$, Giovanni Raspa ${ }^{2}$, Navid Ghavami ${ }^{2}$, Andrea Saracini ${ }^{3}$, \\ Michele Duranti ${ }^{3}$, and Mohammad Ghavami ${ }^{1}$ \\ ${ }^{1}$ School of Engineering, London South Bank University, London, UK \\ ${ }^{2}$ UBT - Umbria Bioengineering Technologies, Spin off of University of Perugia, Perugia, Italy \\ ${ }^{3}$ Department of Diagnostic Imaging, Perugia Hospital, Italy
}

\begin{abstract}
Microwave imaging has received increasing attention in the last decades, in particular for its application in breast lesions detection. Such effort has been encouraged by the fact that, at microwave frequencies, it is possible to distinguish between tissues with different dielectric properties. In such a framework, the Receiver Operating Characteristic (ROC) curves for a novel microwave apparatus for breast lesions detection are empirically determined here through clinical examinations on 22 healthy breasts and on 29 breasts having lesions (including carcinoma, fibroadenoma, and microcalcifications). The apparatus operates in air, with 2 antennas, and uses a Huygens Principle based algorithm to generate images, which are homogeneity maps of tissues' dielectric properties.
\end{abstract}

\section{INTRODUCTION}

Breast cancer is the most prevalent form of cancer in women [1]. Several countries have adopted mass breast cancer screening programs as a way for detecting the disease early and most EU countries are using tools such as mammography machines as the screening methodology. However, mammography has some inconveniences, including:

i) Ionizing radiations. Mammography uses x-rays, which may be dangerous [2];

ii) Mammography is less effective with dense tissue. Mammography is less likely to find breast cancer in young women, who normally have denser breast tissue. Depending on factors such as breast density, mammography may miss up to $30 \%$ of breast cancers in young women [3];

iii) Discomfort. Mammogram equipment applies up to $19 \mathrm{~kg}$ of pressure to the breasts. Not surprisingly, up to $36 \%$ of women refuse to have regular breast screening due to the discomfort and pain [4].

Since the early years of the 21st century, microwave imaging has received increasing attention, in particular for its breast cancer detection applications, encouraged by the considerable difference in the dielectric properties of malignant and normal tissues at microwave frequencies. Specifically, as shown in $[5,6]$, a significant dielectric properties contrast between healthy breast tissue and malignant tissue is present; this contrast is shown to be up to a factor of five in conductivity and permittivity. Meanwhile, newer studies suggest the existence of this contrast only between fatty and malignant breast tissues, and a lower contrast (as low as 10\% in dielectric properties) between healthy fibro glandular and malignant tissues [7-9]. In addition, in [9] it is pointed out that the dielectric properties of benign lesions are similar to the properties of fibro glandular tissues.

Current ongoing research in microwave breast imaging can be separated mainly into microwave tomography and radar-based techniques [10]. Microwave tomography attempts to reconstruct the full electrical profile of the breast by solving a rather ill-posed and nonlinear inverse scattering problem [10]. Complex mathematical formulations causing solution instability is one of the main drawbacks of microwave tomography. On the contrary, radar-based imaging requires the solving of a simpler problem of finding the microwave scattering map based on differences in dielectric properties of the breast tissues; , however, it suffers from low signal to clutter ratio [10]. Few microwave breast imaging prototypes are at clinical trials stage, among which are the prototype developed by the University of Bristol [11] and the prototype developed by the University of Quebec [12].

Recently, a novel microwave apparatus (MammoWave, UBT Srl, Perugia, Italy) for breast lesions detection has been constructed [13]. Such apparatus operates in air, with two antennas, and uses a Huygens Principle (HP) based algorithm to generate images, which are homogeneity maps of the tissues dielectric properties $[14,15]$. Validation of the employed apparatus using clinical data is 
shown in this paper. Specifically, we introduce a metric to measure the non-homogenous behaviour of the image, and the Receiver Operating Characteristic (ROC) curves are empirically determined through clinical examinations on 22 healthy breasts and on 29 breasts having lesions (including carcinoma, fibroadenoma, and microcalcifications).

\section{METHODS AND MATERIALS}

The apparatus is constituted by one transmitting antenna (TX) and by one receiving antenna (RX). Both antennas operate in the frequency band of $1-9 \mathrm{GHz}$. The apparatus is further constituted by: a hub with a cup which is aimed to contain the breast of the patient (prone positioned) and two arms to rotatably associate TX and RX to the hub. The hub and the cup were designed after considering the shape and size of the breast. The transmitting antenna is arranged in a more radially external position than the receiving antenna. The receiving antenna is arranged in a more radially external position than the cup containing the breast (see Fig. 1). Both TX and RX are configured to be rotatably moved around the azimuth, in such a way to pick up reflected electromagnetic field in all the different directions, in a multi-bistatic fashion. Both TX and RX are connected to a Vector Network Analyzer VNA (Copper Mountain Technologies, IN, USA). The apparatus (appropriately integrated in a bed) can be seen in the same Fig. 1.
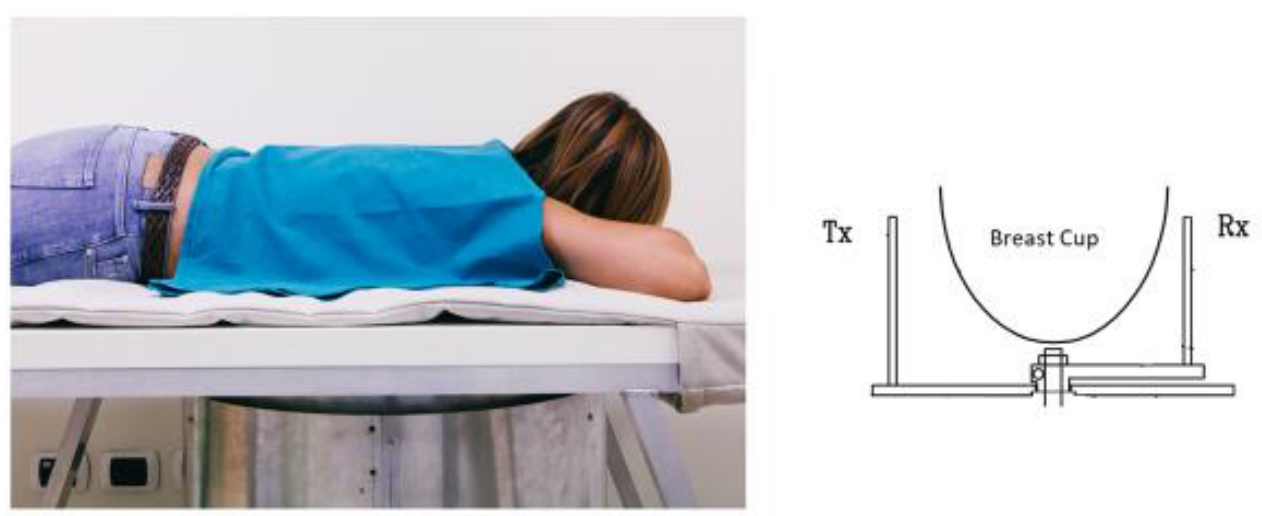

Figure 1. The microwave apparatus consists of a cup that holds the breast when the patient lies prone on the examination table. The transmitting (TX) and receiving (RX) antennas are located inside a hub and can be moved around the azimuth, to irradiate the breast (through TX) and capture the microwaves scattered by the breast itself (through RX).

To generate the image, the signals measured by the receiving antenna, i.e., the complex $S_{21}$ of the VNA, are processed by the processing unit through an imaging algorithm based on HP. In this way, a digital image is generated, as described in $[14,15]$.

Clinical validation on volunteers is currently in progress at Perugia Hospital, Italy. Specifically, the Ethical Committee of Regione Umbria, Italy, approved the clinical validation initially on 45 volunteers (N. 6845/15/AV/DM of 14/10/2015). Recently, Foligno Hospital, Italy, has been authorized by the Ethical Committee of Regione Umbria, Italy, to join the clinical validation, which has been extended to 100 volunteers (N. 10352/17/NCAV of 16/03/2017). The protocol concerns a feasibility study for detection of breast lesions using the proposed microwave mammogram apparatus, with the aim of quantifying the potential of the proposed microwave mammogram apparatus to be used for medical technology screening.

We present the results of the first 51 breasts, each one with the correspondent output of the radiologist study review obtained using echography and/or mammography and/or MRI and/or histological exam (where required). Echography was performed using the MyLab 70 xvg Ultrasound Scanner (Esaote, Genova, Italy); mammography was performed using Selenia LORAD Mammography System (Hologic, Marlborough, MA); magnetic resonance imaging was performed through a 3.0 T MAGNETOM scanner (Siemens Healthcare, Erlangen, Germany). The corresponding output of the radiologist study review has been used as gold standard for classification of the breasts in two categories: healthy (i.e., breasts with no lesion) and non-healthy (i.e., breasts containing lesions). In addition, where possible, the breast type has been classified accordingly to its density, following the scale defined by the American College of Radiology which goes from ACR type A (extremely fatty breast) to ACR type D (extremely heterogeneous fibroglandular breast) [16]. The 
lesion type, if presents, has been classified as carcinoma, fibroadenoma, microcalcifications and other lesions [17-19].

The informed consent was obtained from all subjects. All procedures performed in this study were in accordance with the ethical standards of the institutional and/or national research committee and with the 1964 Helsinki declaration and its later amendments or comparable ethical standards. The research carried out in accordance with the Code of Ethics of the World Medical Association (Declaration of Helsinki) for experiments involving humans.

Microwave images have been obtained using low-power $(1 \mathrm{~mW})$ microwave signals within the band of $1-9 \mathrm{GHz}$. For each microwave image, we calculated the parameter Max/Avg (maximum divided by the average of intensity). Using the gold standard output of the radiologist study review, we calculated: the mean and standard deviation of Max/Avg for the healthy breasts; the mean and standard deviation of Max/Avg for the non-healthy breasts. Unpaired t-test for Max/Avg of the healthy breasts and Max/Avg of the non-healthy breasts has also been performed.

Finally, we empirically evaluated the receiver operating characteristic (ROC) curve using Max/Avg for classification. Specifically, we evaluated True Positive (TP) rate and False Negative (FN) rate as following:

i) we fix a given Threshold for the parameter Max/Avg;

ii) if $\operatorname{Max} / \operatorname{Avg}>$ Threshold, the image is classified as positive, i.e., non-healthy;

iii) if Max/Avg < Threshold, the image is classified as negative, i.e., healthy;

iv) we change the value of the Threshold and we repeat the previous steps.

ROC curve is determined using TP and FN rate values obtained for the different Threshold. ROC curve is also determined for the subset of dense breasts (ACR type C $+\mathrm{D}$ ).

\section{RESULTS AND DISCUSSIONS}

According to the radiologist study review, 22 healthy breasts (16 dense) and 29 non-healthy breasts (21 dense) underwent microwave imaging (which included 7 carcinoma, 9 fibroadenoma, 5 microcalcifications). More details on the population used for this study can be found in Table 1.

As an example, microwave images are given for: heterogeneously dense breast with carcinoma located in the inner quadrants (Fig. 2(a)); scattered fibroglandular healthy breast (Fig. 2(d)). The correspondent mammography images are given in Figs. 2(b), (c) and Figs. 2(e)-(f). Microwave images are homogeneity maps of tissues' dielectric properties, and are given here as $2 \mathrm{D}$ images in the azimuthal plane, i.e., coronal plane. Microwave images are normalized to unitary average of the intensity. $X$ and $Y$ are given in meter. Intensity is given in arbitrary unit, with a scale from 0 to 2 .

Table 1. Patient population used for this study, with some details including the correspondent output of the radiologist study review.

\begin{tabular}{|c|c|c|c|c|c|c|c|}
\hline & $\begin{array}{c}\text { Total } \\
\text { cases } \\
(\mathbf{n})\end{array}$ & $\begin{array}{c}\text { Mean age } \\
(\mathbf{y})\end{array}$ & $\begin{array}{c}\text { Age } \\
\text { range } \\
(\mathbf{y})\end{array}$ & $\begin{array}{c}\text { Carci- } \\
\text { noma }\end{array}$ & $\begin{array}{c}\text { Fibroad- } \\
\text { enoma }\end{array}$ & $\begin{array}{c}\text { Microcalci- } \\
\text { fications }\end{array}$ & $\begin{array}{c}\text { Other } \\
\text { lesions }\end{array}$ \\
\hline $\begin{array}{c}\text { Healthy } \\
\text { breasts }\end{array}$ & 22 & 45 & $29-70$ & - & - & - & - \\
\hline $\begin{array}{c}\text { Non-healthy } \\
\text { breasts }\end{array}$ & 29 & 52 & $20-86$ & 7 & 9 & 5 & 8 \\
\hline
\end{tabular}

Figures 2(g)-(l) show the microwave image given in (Fig. 2(a)) after filtering the intensity through thresholds equal to $1,1.25,1.50,1.80$.

A certain level of inhomogeneity can be seen in both Fig. 2(a) and Fig. 2(d); however, inhomogeneity is more pronounced in Fig. 2(a). A dedicated metric, i.e., Max/Avg, has been introduced to measure the non-homogenous behaviour of the image. For the healthy breasts, mean and standard deviation of Max/Avg are 1.70 and 0.09, respectively; for the non-healthy breasts mean and standard deviation of Max/Avg are 1.82 and 0.11 , respectively.

Unpaired t-test for Max/Avg of the healthy breasts andMax/Avg of the non-healthy breasts leads to a $\mathrm{P}$ value of 0.049 ; by conventional criteria, this difference is considered statistically significant. Different thresholds for Max/Avg, spanning from $(1.70-3 \times 0.09)$ to $(1.70+3 \times 0.09)$, 
have been used to calculate $\mathrm{TP}$ rate and $\mathrm{FN}$ rate, and thus to empirically evaluate ROC curve. ROC curve is determined also for the subset of dense breasts.

ROC curves are given in Fig. 3, from which it follows that Max/Avg of microwave images can be successfully used for classifying breasts containing lesions. In addition, TP rate increases in the dense breasts.

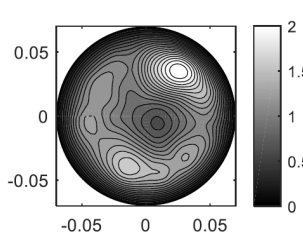

(a)

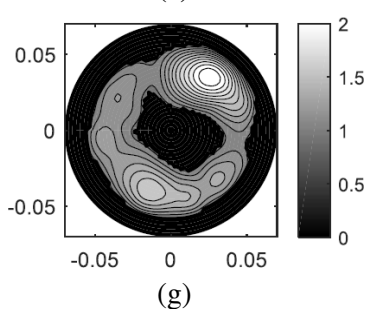

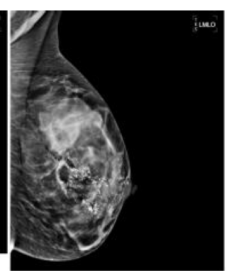

(c)

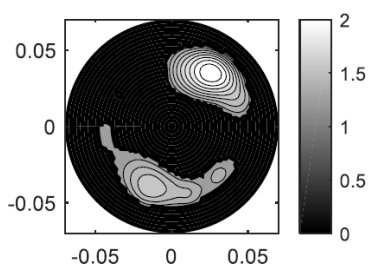

(h)

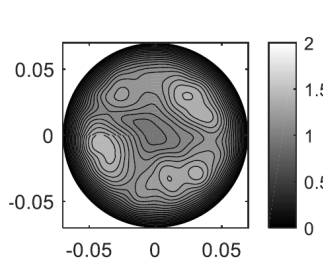

(d)

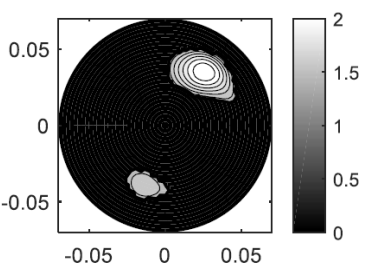

(i)

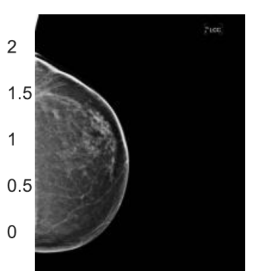

(e)

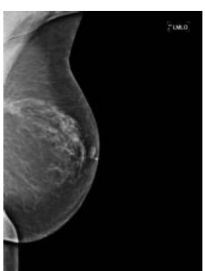

(f)

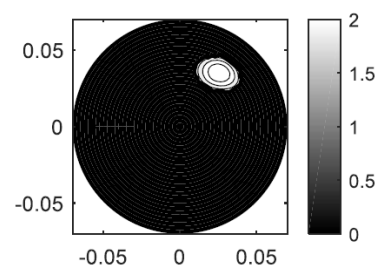

Figure 2. As an example, microwave images are given for: (a) heterogeneously dense breast with carcinoma located in the inner quadrants; (d) scattered fibroglandular healthy breast. The correspondent mammography images are given in (b), (c) and (e), (f). Microwave images are homogeneity maps of tissues' dielectric properties, and are given here as 2D images in the azimuthal plane, i.e., coronal plane. Microwave images are normalized to unitary average of the intensity. $X$ and $Y$ are given in meter. Intensity is given in arbitrary unit, with a scale from 0 to 2. Figs. (g), (h), (i), (l), show the microwave image given in (a) after filtering the intensity through thresholds equal to $1,1.25,1.50,1.80$.

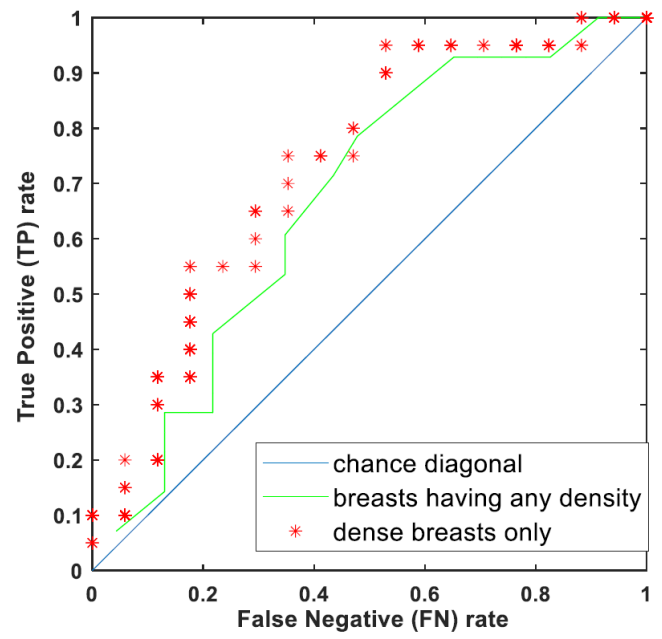

Figure 3. The empirically evaluated receiver operating characteristic (ROC) curve obtained using Max/Avg for classification.

\section{CONCLUSIONS}

Recently, a novel microwave imaging apparatus which resorts to HP has been constructed. The microwave apparatus does not emit ionizing radiation; thus, it is completely safe for the patients. Moreover, no matching liquid is required. Together with its simplicity, HP methodology permits the capturing of the extent to which different tissues, or differing conditions of tissues, can be discriminated, and hence render contrast in the final image. HP allows all the information in the frequency domain to be utilized by combining the information from the individual frequencies to construct a consistent image, which represents a homogeneity map of tissues' dielectric properties. 
A dedicated metric, i.e., Max/Avg, has been introduced to measure the non-homogenous behaviour of the image. It has been show here that Max/Avg can be successfully used for classifying breasts containing lesions.

\section{ACKNOWLEDGMENT}

This project has received funding from the European Union's Horizon 2020 research and innovation programme under grant agreement No. 830265.

\section{REFERENCES}

1. OECD, Mammography machines (indicator), doi: 10.1787/685c9c5e-en, 2019.

2. Miglioretti, D. L., J. Lange, J. J. van den Broek, et al., "Radiation-induced breast cancer incidence and mortality from digital mammography screening: A modeling study," Ann. Intern. Med., Vol. 164, No. 4, 205-14, 2016.

3. Oestreicher, N., C. D. Lehman, D. J. Seger, D. S. M. Buist, and E. White, "The incremental contribution of clinical breast examination to invasive cancer detection in a mammography screening program American," Journal of Roentgenology, Vol. 184, No. 2, 428-432, 2005.

4. Whelehan, P., et al., "The effect of mammography pain on repeat participation in breast cancer screening: A systematic review," The Breast, Vol. 22, No. 4, 389-394, 2013.

5. Li, X. and S. C. Hagness, "A confocal microwave imaging algorithm for breast cancer detection," IEEE Microwave and Wireless Components Letters, Vol. 11, No. 3, 130-132, 2001.

6. Bond, E. J., et al., "Microwave imaging via space-time beamforming for early detection of breast cancer," IEEE Transactions on Antennas and Propagation, Vol. 51, No. 8, 1690-1705, 2003.

7. Nikolova, N. K., "Microwave imaging for breast cancer," IEEE Microwave Magazine, Vol. 12, No. 7, 78-94, 2011.

8. Lazebnik, M., et al., "A large-scale study of the ultrawideband microwave dielectric properties of normal breast tissue obtained from reduction surgeries," Physics in Medicine \& Biology, Vol. 52, No. 10, 2637, 2007.

9. Lazebnik, M., et al., "A large-scale study of the ultrawideband microwave dielectric properties of normal, benign and malignant breast tissues obtained from cancer surgeries," Physics in Medicine \& Biology, Vol. 52, No. 20, 6093, 2007.

10. Conceicao, R. C., J. J. Mohr, and M. O'Halloran, An Introduction to Microwave Imaging for Breast Cancer Detection, Springer, 2016.

11. Preece, A. W., I. Craddock, M. Shere, L. Jones, and H. L. Winton, "MARIA M4: clinical evaluation of a prototype ultrawideband radar scanner for breast cancer detection," Journal of Medical Imaging, Vol. 3, No. 3, 033502, 2016.

12. Li, Y., E. Porter, A. Santorelli, M. Popović, and M. Coates, "Microwave breast cancer detection via cost-sensitive ensemble classifiers: Phantom and patient investigation," Biomedical Signal Processing and Control, Vol. 31, 366-376, 2017.

13. Tiberi, G. and R. Raspa, "Apparatus for testing the integrity of mammary tissues," patent No. 0001413526.

14. Ghavami, N., G. Tiberi, D. J. Edwards, and A. Monorchio, "UWB microwave imaging of objects with canonical shape," IEEE Transactions on Antennas and Propagation, Vol. 60, No. 1, 231-239, Publication Year: 2012.

15. Tiberi, G., et al., "Sensitivity assessment of a microwave apparatus for breast cancer detection," European Congress of Radiology, ECR, C-1390, 10.1594/ecr2018/C-1390, 2018.

16. D’Orsi, C. J., E. A. Sickles, E. B. Mendelson, E. A. Morris, et al., "ACR BI-RADS®Atlas, breast imaging reporting and data system," Reston, VA, American College of Radiology, 2013.

17. Tavassoli, F. A. and P. Devilee, editors, World Health classification of Tumours. Pathology and Genetics of Tumours of the Breast and Female Genital Organs, IARC Press, Lyon, 2003.

18. Lakhani, S., I. O. Ellis, S. J. Schnitt, et al., editors, WHO Classification of Tumour of the Breast, 4th Edition, IARC, Lyon, 2012.

19. Perry, N., M. Broeders, C. de Wolf, S. Törnberg, R. Holland, and L. von Karsa, European Guidelines for Quality Assurance in Breast Cancer Screening and Diagnosis, 4th Edition, European Commission, 2006. 